\title{
ESTUDIANTES UNIVERSITARIOS, POLÍTICA Y MEDIOS DE COMUNICACIÓN EN COSTA RICA. BREVE ANÁLISIS DEL VOTO PRESIDENCIAL, PLATAFORMAS Y MEDIOS TRADICIONALES DE COMUNICACIÓN (2015-2016)
}

\section{UNIVERSITY STUDENTS, POLITICS AND MASS-MEDIA \\ IN COSTA RICA. BRIEF ANALYSIS OF THE PRESIDENTIAL VOTE, PLATFORMS AND MASS-COMMUNICATION (2015-2016)}

\author{
Pablo Carballo Chaves* \\ José Daniel Rodríguez Arrieta**
}

RESUMEN

\begin{abstract}
El presente artículo surge a partir de una investigación de carácter exploratorio que estudia, a través de técnicas cuantitativas, las prácticas políticas de la población universitaria en Costa Rica en general y su percepción sobre la relación entre la política, las plataformas de comunicación y los medios de comunicación. El trabajo se realizó en el marco de los resultados y percepciones de las elecciones nacionales de 2014 y se observó a la televisión como el emisor de información más utilizado por esta población, seguido por el Internet. Asimismo, se plantea la participación política motivada por la misma vivencia universitaria.

PALABRAS CLAVE: POLÍTICA * UNIVERSIDAD * COSTA RICA * PERCEPCIONES * MEDIOS DE COMUNICACIÓN

\section{ABSTRACT}

This article arises from an exploratory research that studies, based on quantitative techniques, the political practices of the university population in Costa Rica and explores their perception of the relationship between politics and the communication platforms in particular. The analysis was done in the context of the results and perceptions of Costa Rica national elections in 2014 and approaches university students who commented about the relationship between their practices, their role in the elections and their perception of the role of the media in this political process motivated by the university context.
\end{abstract}

KEYWORDS: POLITICS * UNIVERSITY * COSTA RICA * PERCEPTIONS * MEDIA

Escuela de Sociología de la Universidad de Costa Rica y Facultad Latinoamericana de Ciencias Sociales, Costa Rica. pcarballo@flacso.or.cr

** Escuela de Ciencias Políticas de la Universidad de Costa Rica y Centro de Investigación y Estudios Políticos de la Universidad de Costa Rica.

josedanielcr@gmail.com 


\section{INTRODUCCIÓN}

Usualmente, se considera a la población universitaria como integrante de un nivel del sistema de educación que se supone crítico e independiente, poseedora de una posibilidad real de acción política e interés en esta. Históricamente, la población estudiantil de educación superior ha supuesto en muchas partes del mundo momentos decisivos con una injerencia directa en la toma de decisiones; precisamente existe considerable bibliografía que ha establecido una relación directa entre nivel educativo y participación política (Murga, 2009, p. 45). El presente artículo genera un acercamiento a esta relación entre población universitaria y política basándose en una reflexión sobre datos cuantitativos, para dar una mirada mayormente descriptiva, exploratoria $y$ especulativa, pero que pueda analizar esta vinculación en el contexto costarricense, con el fin de entender mejor el desempeño político desde un espacio social específico ${ }^{1}$.

A partir de estas relaciones sugeridas, emergen varias interrogantes que buscan ayudar a profundizar en la relación de dicho nivel educativo y las ideas o participación política; en particular, la temática amplia radica en estudiar ¿cuáles son las relaciones entre la población universitaria en Costa Rica y el escenario político-mediático? Asimismo, asumiendo a las personas universitarias como las nuevas generaciones de votantes $y$ posibles tomadoras de decisiones, emerge la significancia social y política de estudiar y reconocer los elementos simbólicos y de prácticas políticas sobre esta población en sus acciones presentes y relacionarles con sus relaciones políticas a futuro.

Se toma en cuenta de Klingemann (2004) dos dimensiones en la participación política:

a) la "convencional" en tanto tres variables básicas: 1) la importancia de la política

$1 \quad$ Una versión preliminar de este artículo se expuso en el V Congreso Internacional en Comunicación Política y Estrategias de Campaña: ¿Está la política escuchando? Un nuevo paradigma, una nueva época ¿Nuevas Prácticas? (ALICE-Asociación Latinoamericana de Investigadores en Campañas Electorales). Buenos Aires, Argentina. Julio de 2016. en la vida de los encuestados, 2) el interés mostrado y 3) la frecuencia con que hablan del tema.

b) la participación "no convencional" basada en cuatro variables: 1) involucramiento en firma de peticiones o demandas, 2) tomar parte activa en manifestaciones callejeras, 3) en boicots y 4) en huelgas no permitidas.

Esto remite a antecedentes que brindan ideas de la trayectoria del fenómeno investigado $y$ que advierten de participaciones convencionales y no convencionales que llenan el espectro de participación política de los estudiantes de educación superior. Para efectos de este estudio se enmarca principalmente en la visión "convencional" de la influencia de los medios de comunicación (o al menos explorando a los medios de comunicación como mecanismos informativos prioritarios en el conocimiento del entorno político), sin embargo, no se deja de lado la incidencia de la no convencionalidad en las perspectivas de la población universitaria.

En este sentido, tal cual lo aporta Ocampo (2010), la universidad es un ente político per se; pero desde luego no en un sentido partidario (aunque se pueda dar tendencias partidarias en diferentes niveles), sino asumiendo la política como la administración de poder en el orden de la función del bien común, lo cual implica una relación directa de la universidad con la sociedad; por ende, de su principal "recurso" - los estudiantes - y la sociedad. Es precisamente a partir de esta relación conceptual que surge el interés y a su vez, la importancia de ubicar las concepciones y percepciones de la población universitaria costarricense como intérpretes y creadores de símbolos sociopolíticos, que al mismo tiempo son origen y explicación de sus acciones u omisiones en la arena política. Ello llevó a elaborar la siguiente pregunta específica que se despliega de la anterior temática amplia indicada $y$ donde surge la reflexión sobre: ¿cuál es la percepción sobre la influencia mediática de los medios de comunicación — televisión e Internet-, sobre la población universitaria costarricense entre los 
18 y 30 años, en el marco del periodo político electoral 2014-2015? ${ }^{2}$

Se pretende brindar aportes cuantitativos en primer término, que se plantean relevantes para la comprensión del fenómeno de la vinculación de la población universitaria a partir de sus propias perspectivas sobre el flujo de información mediática. El presente trabajo parte de información recolectada mayormente durante 2015 y como se verá más adelante, recopila datos acerca de la participación de los universitarios en las elecciones de 2014, así como la participación y percepciones durante el año siguiente. Cabe aportar que hasta la fecha no existe un trabajo investigativo en Costa Rica enfocado exclusivamente en la población universitaria y su quehacer y profundización política, aunque si existen trabajos relacionados con la incidencia $y$ las perspectivas de $y$ sobre la juventud (Ceciliano, 2008) y de la educación en la gestación del desarrollo del país (Solano, 2001). Por otra parte, a nivel internacional existen trabajos importantes en América Latina relacionados con movimientos estudiantiles (Véase: el Observatorio Social de América Latina (osal): Movimientos estudiantiles, 2012), donde se resalta el papel de estos movimientos como estimulantes de la fluidez en la manifestaciones de la colectividad ciudadana.

\section{CONTEXTO POLÍTICO Y LA RELACIÓN CON EL ENTORNO UNIVERSITARIO}

Particularmente en Costa Rica, al igual que en otras latitudes, se ha constatado históricamente un papel activo del sector estudiantil universitario en función de la formulación de demandas y de organización de otros sectores sociales, con el fin de exigir el cumplimiento de derechos o en defensa del Estado social de derecho. Ello se ha hecho evidente en la posibilidad

$2 \quad$ Este artículo se desprende de la investigación denominada: Percepciones y prácticas políticas en la población universitaria de Costa Rica (Proyecto: 01-1683-2016), inscrita en la Escuela de Ciencias Políticas de la Universidad de Costa Rica, a cargo de los investigadores José Daniel Rodríguez Arrieta (Escuela de Ciencias Políticas) y coinvestigador Pablo Carballo Chaves (Escuela de Sociología). de convocatoria, organización y presencia de este sector en las actividades masivas; $y$ en un plano más general, el estudiantado universitario ha ejercido una influencia mediante diversos programas $y$ actividades propias del quehacer universitario - sea investigación, trabajo comunal o proyectos de iniciativa propia- así como en la organización de entes y comunidades para la exigencia de derechos y organización de demandas.

El resultado de las elecciones nacionales presidenciales de Costa Rica en 2014, con la victoria del candidato del Partido Acción Ciudadana (Pac) Luis Guillermo Solís, significó aparentemente el rompimiento con una alternancia de partidos en el poder desde las elecciones de 1982 con la victoria de Luis Alberto Monge, candidato del Partido Liberación Nacional (PLN). A partir de ese momento, las elecciones consiguientes, siete en total, fueron ganadas por ese partido o por su opositor, la Unidad Social Cristiana (pusc), que aglutinó de forma exitosa una oposición ideológica histórica al pLn, pero que nunca había consolidado un movimiento político permanente $y$ estable. Esto muestra una rutina de ejecución de la convencionalidad política que sobre todo ha sido cuestionada desde los espacios universitarios $y$ por diferentes manifestaciones obreras o de izquierda.

Tal como lo plantea Luis P. Solís (2014), la Costa Rica actual es el resultado de una reciente crisis del proyecto neoliberal, lo cual tendría un efecto en el sistema político y sus procesos. Esta crisis del modelo ideológico tiene un impacto en la institucionalidad pública pues:

La atmósfera ética e ideológica impuesta por el predominio neoliberal ha propiciado un juego perverso donde la corrupción se entrevera con políticas ideológicamente motivadas, cuyo fin es restringir los recursos a disposición de las instituciones públicas y debilitar su funcionalidad $y$ capacidad para decidir $y$ actuar (Solís, 2014, p. 79).

Es en este contexto que gana un candidato presidencial más históricamente relacionado a la academia como lo es Luis Guillermo 
Solís ${ }^{3}$. Su trabajo como profesor universitario - a pesar de haber ejercido puestos políticos en el pasado- fue recalcado durante la reciente campaña electoral en relación con un bajo perfil en comparación con los otros candidatos, que a la larga le terminó favoreciendo (Carballo, 2015); de hecho, un número importante de los nombramientos del gabinete fueron personas ligadas igualmente al quehacer académico/universitario.

En esta enmarcación de la política con relación al mundo universitario, se ha vuelto la mirada investigativa hacia la población estudiantil. Cabe recordar que una de las alcaldías más importantes de San José, el cantón de Montes de Oca — cuyo distrito primero es conocido popularmente como "distrito universitario"-, fue ganada por un estudiante de Ciencias Políticas de la Universidad de Costa Rica, Marcel Soler Rubio del PAC ${ }^{4}$. Por esta razón, la importancia de rescatar esta dimensión social del espectro político costarricense, dada la coyuntura de la política nacional.

Como se planteó previamente, en Costa Rica no existen estudios enfocados exclusivamente en la participación política de la población universitaria; por ende, un primer acercamiento a esta población como objeto de estudio, se basó sobre todo en dos elementos básicos: por una parte, la desagregación de datos existentes de investigaciones, censos, encuestas y similares realizados a la totalidad de la población (Raventós, Fournier, Fernández y Alfaro, 2012) o enfocado en población joven, $y$ por otra parte, la recopilación de información

3 Tampoco se puede olvidar que Luis G. Solís fue miembro del Partido Liberación Nacional, lo cual le dio conocimiento práctico y político a lo interno de la lógica de partidos políticos.

En la campaña interna del Partido Liberación Nacional del 2017 (aunque se vio incipientemente en el inicio electoral en otros partidos) se pudo apreciar la alta incidencia de dos circunstancias: a) el uso de herramientas tecnocomunicacionales en forma muy amplificada; $y$ relacionado a esto b) la presencia de jóvenes como participantes (tanto a cargos de elección, como dentro del trabajo de base) en donde figuraban estudiantes universitarios gestionando dinámicas políticas en dichos partidos. relativa a la praxis política en diferentes actividades relativas a la incidencia social o política (los datos del Tribunal Supremo de Elecciones o las acciones colectivas, Mora, 2008). De lo anterior se rescata cuatro ámbitos concretos de participación política de la población joven general, no especificado por su vinculación universitaria:

$\diamond \quad$ La participación en diversas manifestaciones $y$ protestas relacionadas con temas políticos a mediana y gran escala en diversas partes del país.

$\diamond \quad$ Apoyo a otros movimientos sociales, particularmente en casos conflictivos en zona rural.

$\diamond \quad$ Involucramiento directo en la estructura formal de partidos políticos de todas las tendencias.

$\diamond \quad$ Organización de actividades informativas $y$ de debate sobre temas políticos de diversa índole.

A partir de estas acciones, las estudiantes $y$ los estudiantes universitarios han gestionado su integración en el ámbito político en Costa Rica, relacionándose en gran parte con la defensa del Estado social de derecho y el respaldo a las poblaciones vulnerables. Casos más recientes han sido las movilizaciones respecto a lo que se llamó el "Combo ice" en 2000 y la más reciente oposición al Tratado de Libre Comercio (TLC) que culminó con un referéndum en 2007 (Escalona, 2012). Ambos con tonos de discusión política respecto a modelos de aperturismo comercial relacionados con procesos de neoliberalización en América Latina, principalmente vinculado con el mismo entorno educativo y sus referencias a la universalidad del acceso.

Asimismo, es importante rescatar, como lo han hecho numerosas investigaciones, que la población con mayor grado de estudios tiende a ser más activa en relación a la participación cívica o política (Murga, 2009; Pignataro y Treminio, 2015). De hecho, esta variable tiende a ser más determinante para la participación política que cualquier otra, lo cual alerta $y$ da pie para seguir sobre la reflexión acerca de una población que tiene diferentes posibilidades $y$ 
manifestaciones sociopolíticas con alcances sociales. Respecto a estudios asociados a la campaña presidencial reciente (2014) y medios de comunicación, se puede ver el trabajo de Nicolas, Alvarado, Rodríguez y Abarca (2015) quienes exploran la presentación que se hizo en diferentes medios de comunicación (enfocados en prensa escrita) sobre programas, ideas y elementos asociados a los partidos políticos bajo la denominación de "promesas políticas".

\section{ABORDAJE METODOLÓGICO Y CARACTERIZACIÓN GENERAL DE LA INFORMACIÓN}

Con el objetivo de explorar la relación entre consideraciones sobre la política y la población universitaria costarricense se construyó un instrumento estadístico que permitió recolectar respuestas de la población escogida. Esto debido a que el carácter de la investigación sigue un enfoque prioritariamente cuantitativo. El acercamiento fue por medio de un sondeo, por lo que no se contó con una muestra representativa. Es importante afirmar que la lógica de la recopilación de la muestra fue de carácter no probabilístico, que presenta las circunstancias de la selección a conveniencia (Gómez, 2012), es decir, que la escogencia no brindó las misma oportunidad de selección a todo el grupo universitario. Esto se debió en primer lugar por la accesibilidad de recursos para gestionar una muestra más compleja $y$ probabilísticamente representativa. Así, el total de la población universitaria del país para el año 2015, fue alrededor de 208 mil personas ${ }^{5}$ - matrícula en el primer período lectivo- (Estado de la Educación, 2015, p. 192), comprendiendo solamente la suma de estudiantes de las universidades públicas y privadas, la muestra fue de 409 personas.

5 La muestra representativa (n) que se buscaba obtener era de: 665 (con 99\% de confianza y un nivel de error de 5\%). Se tomó la decisión de un sondeo (aun cuando se podía bajar el nivel de confianza para obtener una muestra representativa), porque también se presentaron dificultades en la aleatoriedad, debido a la sobre presencia de personas de Ciencias Sociales y de universidades públicas, entre otras posibles sesgos (Gómez, 2012).
La información fue recolectada en los meses de setiembre y octubre de 2015. Consistió en la combinación de dos formatos: 1) Cara a cara —es decir, encuesta tradicional-, y 2) Vía virtual - por medio de encuesta digital-. La Tabla 1 expone algunas de las características de cada formato y el proceso de elaboración de la base de datos.

TABLA 1

CARACTERÍSTICAS DE LOS FORMATOS DE RECOLECCIÓN DE INFORMACIÓN

\begin{tabular}{|c|c|c|}
\hline CARACTERÍSTICAS & $\begin{array}{c}1) \\
\text { CARA } \\
\text { A CARA }\end{array}$ & $\begin{array}{c}2) \\
\text { VÍA } \\
\text { VIRTUAL }\end{array}$ \\
\hline Formato & $\begin{array}{c}\text { Boleta } \\
\text { tradicional }\end{array}$ & $\begin{array}{l}\text { Boleta } \\
\text { digital }\end{array}$ \\
\hline Cantidad & 200 & 209 \\
\hline $\begin{array}{l}\text { Meses de } \\
\text { recolección }\end{array}$ & $\begin{array}{c}\text { septiembre- } \\
\text { octubre }\end{array}$ & $\begin{array}{c}\text { septiembre- } \\
\text { octubre }\end{array}$ \\
\hline $\begin{array}{l}\text { Programas de } \\
\text { procesamiento }\end{array}$ & SPSS & $\begin{array}{c}\text { DRIVE*-Excel- } \\
\text { SPSS }\end{array}$ \\
\hline
\end{tabular}

*DRIVE, programa digital que se facilita por medio de GMAILGoogle para captar información digital.

Nota: La integración final de la base de datos se hizo en el spss.

Fuente: Elaboración propia.

Componer la información en dos fases tiene tanto una razón pedagógica ${ }^{6}$, así como, la posibilidad de ampliar la muestra no representativa (sondeo) y lograr llegar a personas en otras zonas no centralizadas del país. El rango de edad comprendido de personas encuestadas fue de 19 a 30 años, pero donde el principal rango fue el más joven 19-21 años (gráfico 1).

$6 \quad$ La razón pedagógica es debido a que la temática y recolección de la información fue pensada y hecha con apoyo del grupo de estudiantes del curso: Técnicas de manejo de la información: grupo.001. Prof. Pablo Carballo Chaves (II Semestre/ 2015). Escuela de Sociología de la Universidad de Costa Rica. Debido a esto es que la práctica consistió parcialmente en la recolección tanto presencial como virtual de la información. 


\section{GRÁFICO 1 \\ EDADES INDICADAS POR LAS PERSONAS ENCUESTADAS \\ DATOS PORCENTUALES, UNIVERSITARIOS, 2015}

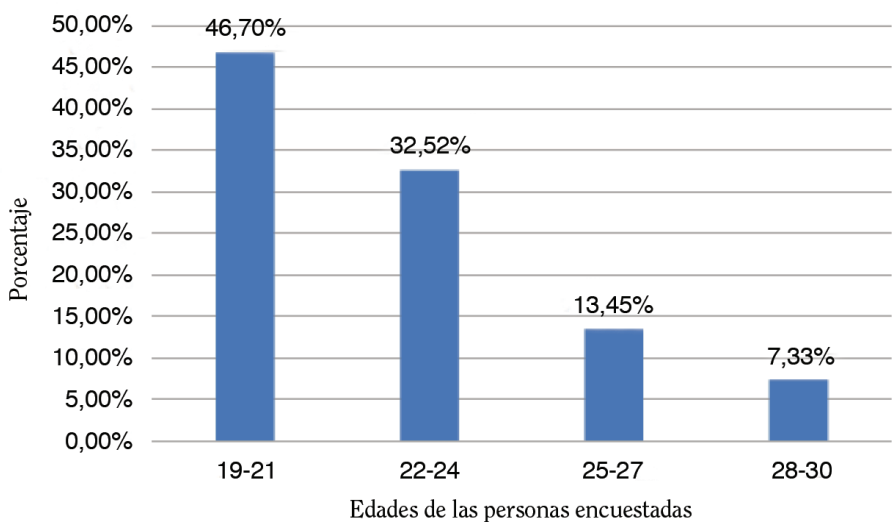

Fuente: Elaboración propia.

Los gráficos 1 y 2 , y los cuadros 1, 2 y 3 , presentan la información descriptiva de la base de datos creada, a partir de un total de 409 personas registradas y que respondieron a $60 \mathrm{va}-$ riables. El gráfico 1 y el cuadro 1 presentan la información por edades y por lugar de procedencia registrada. En estos se observa que la concentra- ción de las personas analizadas se presenta en la zona central (GAm) Gran Área Metropolitana, personas comprendidas en las edades entre los 19 y 24 años. Las edades donde menos se registró información fueron entre los 25 y los 30 años. En el caso de la distribución por género se observa una casi paridad en las personas encuestadas.

\section{GRÁFICO 2 \\ GÉNERO INDICADO DE LA POBLACIÓN UNIVERSITARIA ENCUESTADA \\ DATOS PORCENTUALES \\ 2015}

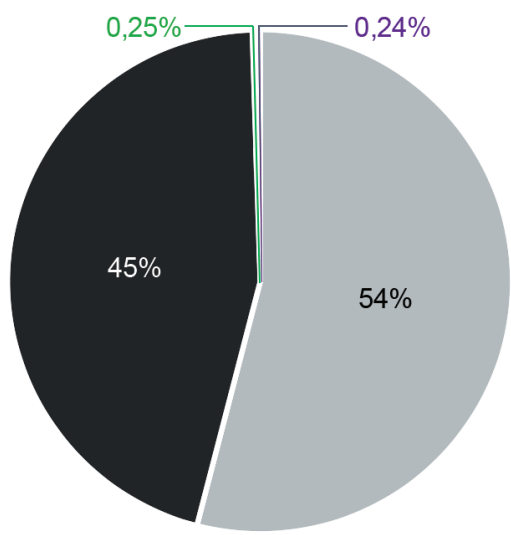

Femenino

Masculino

- Transgénero $(0,25 \%)$ Ns/Nr $(0,24 \%)$

Fuente: Elaboración propia. 
El Gráfico 2 expone esta división con el objetivo de abarcar las nuevas dimensiones valorativas con que las personas (en alguna medida más jóvenes) empiezan a reidentificarse como sería la dimensión de las personas transgénero. Aunque en términos porcentuales no generaron consideraciones de peso al trabajar con las categorías regulares de hombre (45\%) y mujer (54\%), las mujeres presentan 10 puntos porcentuales más en el sondeo.

Respecto a la orientación sexual, la población que se denominó heterosexual fue de un $77 \%$, mientras que la población autodenominada homosexual fue de un $12 \%$. Dejando alrededor de un $11 \%$ que se denominó de alguna otra forma: como bisexual en un $7 \%$, asexual en poco más de un $1 \%$ y un "otros" de alrededor de un 2\%. La información de género y orientación sexual buscan dar una contextualización de la población estudiada, pero no tiene una reflexión desarrollada en este documento.

La desagregación por provincia según la muestra se hace en el cuadro 1, donde San José fue el bloque de trabajo principal de los datos, obteniendo casi el 50\% del volumen de las personas encuestadas.

CUADRO 1

DISTRIBUCIÓN DE LAS PERSONAS

ENCUESTADAS SEGÚN PROVINCIA 2015

\begin{tabular}{lcc}
\hline PROVINCIA & ABSOLUTOS & PORCENTAJES \\
\hline San José & 197 & 48,2 \\
Alajuela & 57 & 13,9 \\
Heredia & 39 & 9,5 \\
Cartago & 74 & 18,1 \\
Puntarenas & 9 & 2,2 \\
Guanacaste & 17 & 4,2 \\
Limón & 7 & 1,7 \\
(Extranjero/a) & 9 & 2,2 \\
\hline TOTAL & 409 & 100 \\
\hline
\end{tabular}

Fuente: Elaboración propia.
El Cuadro 2 presenta la información recolectada según la pertenencia a universidades públicas o privadas. Puede verse que el $78 \%$ está en las universidades públicas y el $22 \%$ en universidades privadas (no se especifica por universidad, sino por su ubicación en el espectro público-privado ${ }^{7}$ ). La idea de trabajar por tipo de universidad es eventualmente poder ahondar en la diversidad $y$ posicionamiento político, según en qué área se encuentren estudiando las personas encuestadas. Así también, explorar una dimensión mayor de opiniones respecto a las ideas políticas. Por esa razón, se tomó en cuenta los años de estudio que la población encuestada lleva en la universidad. De manera general, la población que se encuentra entre 1 y 2 años en la universidad es alrededor de un 28\%. Mientras que los que tienen 3 o más años en la universidad, para efectos del sondeo presentado, es de alrededor de un $71 \%$. Esto permite tener algún acercamiento con una población que ya ha tenido bagaje o relación con temas políticos de alguna forma, debido a su estancia en el período universitario.

CUADRO 2

UNIVERSIDADES EN LAS QUE SE ENCUENTRAN MATRICULADAS LAS PERSONAS ENCUESTADAS 2015

\begin{tabular}{lcc}
\hline $\begin{array}{l}\text { UNIVERSIDAD / } \\
\text { MATRICULA }\end{array}$ & ABSOLUTOS & PORCENTAJES \\
\hline Universidad Pública & 319 & 78 \\
Universidad Privada & 90 & 22 \\
\hline TOTAL & 409 & 100 \\
\hline
\end{tabular}

* Algunos estudiantes pueden estar matriculados en varias universidades, por lo que se registró la universidad que indicaron como única o central.

Fuente: Elaboración propia.

$\overline{7} \quad$ Se debe considerar que un estudiante universitario puede estar en varias universidades a la vez (tanto en varias privadas o púbicas, como una en cada dimensión). Por lo que se tomó la decisión de solo contabilizar una, según la respuesta que dieran a la pregunta sobre si ¿̇su universidad (única o principal) es pública o privada? 
El desarrollo de la metodología se formuló en relación con el acceso a una pluralidad de personas en el entorno universitario, que se puede ver desde la dicotomía referida como lo público frente a lo privado, así como a las inequidades respecto a zonas rurales y urbanas. Sin embargo, uno de los ejes de principal interés analítico que se busca desarrollar, consiste en la dimensión sobre la influencia mediática o las valoraciones sobre los medios de comunicación $y$ la población universitaria en el contexto político electoral. El Cuadro 3 muestra que la población estudiada señaló que la televisión (66\%) y el Internet (27\%) son las principales plataformas mediáticas que influyeron en la decisión de voto en las elecciones de 2014. Entre las razones para esto, está el uso de la tecnología en población relativamente joven y el contexto universitario que fomenta este acceso tecnológico. Asimismo, se ha gestado un mundo donde lo tecnológicovisual-virtual es parte de la forma de concebir la vida en la población universitaria.

CUADRO 3

PLATAFORMAS MEDIÁTICAS QUE SE CONSIDERA INFLUYÓ MÁS EN LA DECISIÓN DE VOTO 2015

\begin{tabular}{lcc}
\hline MASS MEDIA & FRECUENCIA & PORCENTAJES \\
\hline Televisión & 271 & 66,3 \\
Internet & 112 & 27,4 \\
Prensa & 9 & 2,2 \\
Otros & 17 & 4,1 \\
\hline TOTAL & 409 & 100 \\
\hline
\end{tabular}

Fuente: Elaboración propia.

De lo anterior se destaca que la población en estudio indicó que durante la primera ronda, un $82 \%$ sí votó, mientras que casi un $15 \%$ no votó (con un 3,2\% de $\mathrm{NS} / \mathrm{NR}$ ). En el caso de la segunda ronda, los que sí votaron fueron $68 \%$, mientras que alrededor de un $27 \%$ no votó (con un $4 \%$ que indicó Ns/NR y un $0,5 \%$ de datos perdidos). Se observa que para la segunda ronda disminuyeron en un $14 \%$ aquellos que sí votaron en la primera ronda. Esto permite cerrar con un panorama general de la recopilación del material cuantitativo $y$ que permite centrarse en la pregunta principal que advierte de la relación de la población universitaria, política convencional (elecciones) y su relación con los medios de comunicación.

\section{ANÁLISIS DE LA RELACIÓN ENTRE POLÍTICA Y MEDIOS DE COMUNICACIÓN}

El análisis se presenta a partir del posicionamiento electoral de la población universitaria en la última elección en Costa Rica (2014). Esto brinda una idea del acercamiento a los temas institucionales en materia de política formal representativa. En un segundo momento se explora la importancia que las personas sondeadas le dan a las plataformas mediáticas (es decir, espectros de medios de comunicación masiva) en la influencia sobre lo político y sobre todo, en el proceso de votación. Por último, se realiza una reflexión puntual sobre dos de los medios de comunicación (uno televisivo y otro impreso) que más impactaron en el escenario político del país. Con este último rubro de análisis se busca presentar ejemplos de la influencia que ciertos medios tienen sobre la población universitaria (y en general también), tanto por su presencia como por la readaptación de acceso de comunicación debido a los cambios comunicacionales.

\section{VOTO EN LA PRIMERA Y SEGUNDA RONDA: ESTUDIANTES UNIVERSITARIOS}

En 2014, el proceso electoral tuvo por segunda vez en la historia contemporánea costarricense una segunda ronda, esto en los últimos 60 años. Esta vez el partido tradicional más fuerte, el Partido Liberación Nacional (PLN), fue vencido por uno de los partidos más recientes, el Partido Acción Ciudadana (PAc), pero con alrededor de 10 años en la palestra político electoral. Esta situación tuvo su contexto en un escenario de insatisfacción social y política que, entre otros elementos, se decantaron en la población por un voto que quebrara con una tradición partidista ${ }^{8}$. Esta situación hace

$8 \quad$ Para profundizar en el fenómeno electoral discursivo véase: Carballo, 2015. Para explorar ideas sobre las tendencias y fracturas sociopolíticas véase: Carballo, 2016. 
constar que es importante posicionar también a la población universitaria en dicho escenario electoral reciente en Costa Rica. Es decir, una gran parte de la incidencia en la crítica se fomentaba en ciertas esferas universitarias, desde las cuales los movimientos estudiantiles se manifestaban en contra de una visión partidaria tradicional. Los mismos partidos Pac $y$ Frente Amplio surgieron en buena medida en espacios con una presencia universitaria, tanto de funcionarios como de grupos $y$ personas en el plano estudiantil.

Como se indicó anteriormente, es fundamental establecer una ubicación de la población universitaria estudiada respecto a su comportamiento en las pasadas votaciones, con el fin de valorar el acercamiento o interés en los procesos políticos, particularmente los considerados formales o convencionales (Klingemann, 2004). En este sentido, el Cuadro 4 presenta cómo votó el estudiantado universitario en la primera y en la segunda ronda. Es importante resaltar que las consideraciones respecto a cada ronda de votación alertan de intereses y comportamientos sociales y políticos distintos. Así, mientras en la primera ronda se expresa más la pluralidad partidista; en la segunda ronda se puede detectar el interés más allá de lo partidista (o menos partidista), así como una posible desavenencia social hacia la dinámica electoral representativa $y$ principalmente, una perspectiva de reproche hacia los partidos tradicionales.

\section{CUADRO 4 \\ VOTO EN PRIMERA O SEGUNDA RONDA \\ POBLACIÓN ESTUDIANTIL UNIVERSITARIA EN COSTA RICA \\ 2015}

\begin{tabular}{lcccc}
\hline & \multicolumn{2}{c}{ PRIMERA RONDA } & \multicolumn{2}{c}{ SEGUNDA RONDA } \\
\hline VOTO & FRECUENCIA & PORCENTAJE & FRECUENCIA & PORCENTAJE \\
\hline Sí & 336 & 82,2 & 278 & 68,3 \\
No & 50 & 14,7 & 112 & 27,5 \\
NS/NR & 13 & 3,1 & 17 & 4,2 \\
\hline TOTAL & 409 & 100 & $407^{*}$ & 100 \\
\hline
\end{tabular}

* Dos datos fueron perdidos en el levantamiento del material.

Fuente: Elaboración propia.

Como se aprecia en el Cuadro 4, el 82\% de los estudiantes sondeados indicaron que sí votaron en la primera ronda, pudiendo indicar su interés por el proceso electoral $y / 0$ por un partido o candidato ${ }^{9}$. La elección se realizó el

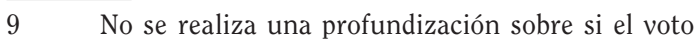
fue nulo o en blanco. Solamente se precisa su votación efectiva de si fue a las urnas o no. Además, para efectos del análisis especulativo que se quiere realizar se tomó la respuesta de un Sí como un voto sobre un candidato específico; y el No como ausencia de las urnas. Esta decisión se realizó porque el interés de la investigación en la que se enmarca el estudio versó sobre las tendencias generales y no en procesos más minuciosos del sistema electoral.
5 de febrero de 2014 y en ese momento los tres partidos que más votos obtuvieron según el Tribunal de Elecciones de Costa Rica fueron ${ }^{10}$ : PAC (31\%), pLN (29,5\%) y Frente Amplio (17,1\%). Es importante rescatar que figuras clave de los miembros del pac fueron miembros del pLn, así como, trabajadores del espacio académico universitario. Además, en términos del abstencionismo en la primera ronda, este fue de un $31,7 \%$ del padrón efectivo de voto (TSE-Costa Rica, 2014).

10 Para que se presente la situación de una segunda ronda, ninguno de los partidos que están participando en la elección debe haber alcanzado un mínimo de $40 \%$ de los votos válidamente emitidos (Constitución Política de Costa Rica. Artículo 138). 
En el caso del sondeo realizado para este estudio, el abstencionismo fue de 14,7\% (no se incluye ns/ NR, pues la persona pudo haber votado, pero no recuerda o no quiso afirmarlo), mostrando un abstencionismo más bajo que en el plano nacional, prácticamente en la mitad.

En el caso de la segunda ronda, la elección se dio entre los dos partidos que más votos sacaron en la primera ronda, PAC $y$ PLN. En esta segunda ronda, el PAc volvió a ganar, pero esta vez con una abrumadora ventaja de $78 \%$ de los que sí votaron y un $22 \%$ para el PLn según los datos oficiales del TSE. Pero en términos de la abstención esta fue de 43\% (casi la mitad del padrón), lo que deja solo un $57 \%$ efectivo de voto del padrón electoral (TSE).

Según los datos de este estudio, en la segunda ronda la abstención también subió casi en el doble de la primera ronda, ahora pasó a ser casi un $28 \%$. Se puede observar que el comportamiento del voto en la población universitaria en la segunda ronda también emula el proceso de resultado general de la población costarricense al subir dicho abstencionismo. El abstencionismo es más grande en el plano nacional y más reducido en el mundo estudiantil universitario (en el Gráfico 3 se muestra la dinámica universitaria respecto a la abstención), pero ambos tienden a duplicarse entre rondas.

GRÁFICO 3

ABSTENCIÓN UNIVERSITARIA ENTRE LA PRIMERA Y SEGUNDA RONDA

DE LA ELECCIÓN DE 2014

(SEGÚN SONDEO DE 2015*)

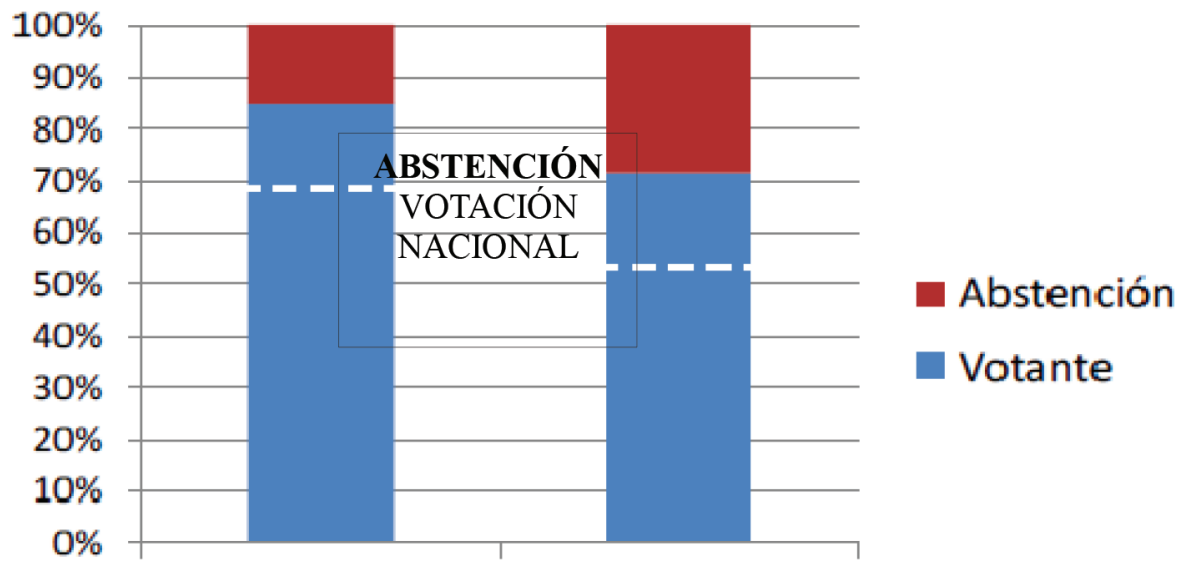

Primera Ronda Segunda Ronda

* No se contabilizan los datos NS/NR.

** Datos del abstencionismo nacional en líneas blancas discontinuas.

Fuente: Elaboración propia con datos de TSE, 2014.

Tres argumentos permiten valorar estos procesos, en primera instancia de cuotas más elevadas de votación en el universo universitario y en segundo lugar, la abstención similar a los datos nacionales. El primer argumento explicativo es que la mayor cuota de voto, tanto en primera como en la segunda ronda, en el caso de la población universitaria remite a que la mayoría son personas jóvenes ubicadas en edades comprendidas entre 19 y 24 años (casi un $80 \%$ de la muestra). En este sentido, se infiere que están iniciando y desarrollando sus prácticas políticas, lo cual indica sus acercamientos o arraigos políticos, haciéndolo en el marco de la universidad y los elementos políticos que en esta se estimulan. Además, 
el estímulo político que se presenta en el entorno universitario funciona también como un impulsor para la praxis política diversa (existente o creando nichos para ello), que va desde lo partidario hasta otras prácticas políticas que no necesariamente son en instancias de partidos, sino en movimientos sociales o de otra denominación específica.

El segundo argumento consiste en una posible variable a contemplar en la población joven, la cual se enfoca en la posibilidad de que sea su primera votación o sus primeras votaciones, por lo cual hay una dinámica de novedad, que puede estar estimulando el proceso. Si bien, esta condición está sujeta al estimulo familiar o de grupos, es importante tenerla presente, para no generar una visión endógena exclusiva para el contexto universitario como razón única para un proceso de acercamiento político, sea convencional o no convencional.

Por último, el tercer argumento consiste en la relación con el entorno mediático. Este participa del mismo entorno de credibilidad y de presión social sobre las consideraciones y apoyos políticos. Lo reflejado en los medios termina por ser parte del marco de referencia que se construye en la bitácora política. Para profundizar en esta relación, las siguientes dos secciones exploran la relación entre plataformas y credibilidad en política.

\section{PLATAFORMAS MEDIÁTICAS E INFLUENCIA POLÍTICA}

Se abordó la relación entre la influencia política por parte de las plataformas de comunicación, entendiendo estas como dinámicas tecnológicas específicas de los medios de comunicación (televisión, prensa, radio, internet, etc.). Según las personas sondeadas, la decisión final de voto tuvo su principal influencia mediática en la televisión, seguido del Internet ${ }^{11}$; es decir,

$11 \quad$ Se debe aclarar que en sentido estricto, la televisión $y$ el Internet no son plataformas de comunicación equiparables estrictamente por sus diferencias tecnológicas. Pero para efectos de estudio establecemos que son plataformas distintas en el sentido de que son dimensiones de la comunicación diferentes una limitada y tradicional (televisión) y otra se preguntó por el espacio o plataforma que más influenciaba en el entorno sociopolítico general en la decisión de voto. Como se observa en el Cuadro $5^{12}$, según datos de la muestra, la televisión obtuvo el más alto dato con un $66,3 \%$, mientras que el Internet fue de $27,4 \%$.

\begin{tabular}{lcc}
$c$ & CUADRO 5 \\
PLATAFORMAS MEDIÁTICAS QUE SE \\
CONSIDERAN CON MÁS INFLUENCIA EN \\
LA DECISIÓN DE VOTO COSTARRICENSE SEGÚN \\
LAS PERSONAS SONDEADAS \\
2015 \\
\multicolumn{4}{c}{ FRECUENCIA } & PORCENTAJE \\
\hline PLATAFORMAS & 271 & 66,3 \\
MEDIÁTICAS & 112 & 27,4 \\
\hline Televisión & 9 & 2,2 \\
Internet & 9 & 2,2 \\
Periódicos & 7 & 1,7 \\
Ninguno & 1 & 0,2 \\
Radio & 409 & 100 \\
NS/NR & & \\
\hline TOTAL & & \\
\hline
\end{tabular}

Fuente: Elaboración propia.

Lo anterior es previsible pues todavía es la televisión la que se mantiene con una percepción de fuerte posicionamiento en la población y sigue siendo un medio de alta credibilidad, además de que su cobertura tiene un peso importante. $\mathrm{Al}$ respecto, de acuerdo con el Instituto Costarricense de Estadística y Censos (INEC), 97,1\% de la población costarricense tiene al menos un televisor en su casa (2016), lo cual implica una alta exposición a cualquier

no limitada (abierta a la interacción) y no tradicional (Internet).

12 Es importante tener presente que la pregunta que fundamenta este cuadro fue acerca de cuál es la plataforma que la población estudiantil universitaria creen que más influyó en la decisión de voto nacional. Sin embargo, en relación con preguntas similares, se detectó que la valoración es en apariencia, coincidente con sus propios criterios, es decir, equivale para comprender la confianza personal sobre dichas plataformas. 
información relativa a las elecciones. A esto se suma lo fácilmente digeribles que son algunos mensajes en el recurso audiovisual.

Asimismo, aunque no se cuenta con datos específicos debido a la reserva de los medios de comunicación, se conoce que la facturación más alta de pautas políticas se paga a las televisoras; esto en sí es un indicador que relaciona la apuesta partidaria y la percepción sobre la exposición y la potencial influencia en el electorado. Además, está en relación directa con la complejización de los procesos publicitarios desde la década de 1980 (Caro, 1994).

Cabe recordar que, de acuerdo con una encuesta del Centro de Investigación y Estudios Políticos (сіEP), la televisión es el medio privilegiado por la población costarricense para informarse con una preferencia del 90,2\% (Villalobos, 2016). Este dato refuerza la percepción de los estudiantes universitarios sondeados y el peso de este medio en términos electorales. Así, la conexión entre acceso nacional, facilidad o simplicidad de mensajes, publicidad especializada y privilegios de legitimación política hacen que la televisión tenga, tanto para la población en general como para la población universitaria, una significativa influencia política.

Al respecto, la mayoría de los sondeados considera que se privilegia, en un sentido amplio, la agenda mediática, particularmente la de la industria televisiva, la cual influencia de mejor forma la agenda pública. Precisamente, Shaw citado por D’Adamo et ál. (2000) señaló que:

La gente tiende a incluir o a excluir de sus propios conocimientos lo que los medios de comunicación incluyen o excluyen de su propio contenido. El público, además, tiende a conceder a las cosas una importancia que refleja el énfasis atribuido por los medios de comunicación a los acontecimientos, a los problemas, a las personas (p.48).

Como se adelantó, en segundo lugar, el medio considerado por los estudiantes universitarios como influyente en el voto de la población costarricense fue Internet. En este aspecto, la población sondeada, mayormente joven, lo recalca desde su cotidianidad; es decir, es una población relacionada habitualmente con Internet y con las redes sociales, por lo que dicho medio está presente de forma constante desde lo personal, laboral y académico.

De acuerdo con datos recientes, Internet es accesado por un $51 \%$ de la ciudadanía costarricense (Araya, 2016) y esta estadística va en ascenso (Jiménez, 2013; Villalobos, 2016). Sobre esto cabe apuntar que las redes sociales como una fuente de información aumenta cada vez entre quienes acceden a Internet. Precisamente cabe recordar que:

El Internet vino a romper parcial y temporalmente el poderío de las frecuencias de radio y televisión, así como el fuerte de la impresión y distribución. Primero fueron las web de noticias, luego los blogs, las revistas y finalmente las redes sociales, con la ayuda del "mailing" (envío de correos) y el Rss (actualización social inmediata), los que permitieron a los medios llegar a públicos amplios (Jiménez, 2013).

Asimismo, a pesar de que, como concuerdan muchos especialistas, en Costa Rica la explotación de las redes sociales en particular, como el Internet en general, es incipiente desde el punto de vista de las campañas electorales y los partidos políticos, su característica de constante auge y cobertura, así como, su bajo costo, ha ido influyendo en su uso por parte de los candidatos, tal como ocurrió en el proceso para la elección de 2014 (Salas, 2013). Los medios tradicionales las utilizan como una fuente no generalizable para medir el pulso político entre los candidatos de turno. Se puede introducir la idea de que esta posibilidad está estableciendo un nexo televisión-Internet como un paradigma de influencia básica que está calando en la población que no solo tiene acceso, sino que también puede producir material en este y publicarlo masivamente.

El Internet, de acuerdo con las y los sondeados, es por sí mismo una plataforma de información más allá del medio digital en particular que se accese (lo cual va ligado a la información que circule por redes sociales). Es decir, las personas recuerdan con más claridad 
que vieron una información en Internet y poco recuerdan en cual medio específico. De acuerdo con Jiménez (2013), para ese año ya existían tres medios digitales de noticias entre las 100 páginas más visitadas de Costa Rica. Aquí el punto es que Internet es la nueva arena política clave y la población que está usando dicho escenario con mayor frecuencia $y$ naturalidad es la juventud, esto a partir de un tridente crucial: acceso/regularidad/necesidad. Esto plantea la preponderancia de una importante mirada crítica $^{13}$ al respecto.

\section{INFLUENCIA, MEDIOS Y CONFIABILIDAD}

Se tomó la decisión de trabajar dos medios de comunicación específicos y particularmente tradicionales, para profundizar en algunos elementos de confiabilidad respecto de las y los universitarios sobre dicha producción noticiosa. Los medios fueron el periódico $L a$ Nación y el telediario: Teletica Canal 7.

Con respecto a los medios de comunicación tradicional, en específico la prensa escrita, un $67,3 \%$ de los estudiantes universitarios encuestados opinaron que La Nación es suficientemente confiable o bastante confiable como fuente de información. Es importante recordar que este medio, a pesar del gran impacto de las redes sociales $y$ el Internet, se ha mantenido como un medio importante de prensa escrita en lo que respecta al tema de noticias e información. Cabe recordar que su público meta es "clase media y media-alta" con estudios universitarios.

Asimismo, el Cuadro 6 evidencia que a medida que aumenta la escala de confiabilidad, la percepción positiva de los estudiantes sondeados disminuye respecto a La Nación. Cuando se analiza este fenómeno de forma desagregada, se observa la misma tendencia tanto para los estudiantes de las universidades privadas como de las universidades públicas. Con respecto a los datos individuales desagregados, se destaca que los estudiantes de las universidades públicas se ubicaron mayormente en una "suficiente confiabilidad" con un 26,4\% y los estudiantes de las universidades privadas se ubicaron en esta variable con un 9,8\%, representando en ambos casos los datos más altos en esta escala.

\section{CUADRO 6 \\ CONFIABILIDAD DEL MEDIO DE PRENSA \\ LA NACIÓN SEGÚN LA PERCEPCIÓN \\ DE LA POBLACIÓN ESTUDIANTIL UNIVERSITARIA SONDEADA 2015}

\begin{tabular}{lcccccc}
\hline & \multicolumn{7}{c}{ PORCENTAJE } \\
\hline UNIVERSIDAD & NADA & POCO & SUFICIENTE & BASTANTE & NS/NR & TOTAL \\
U. Pública & 11,2 & 14,7 & 26,4 & 25,2 & 0,5 & 78 \\
U. Privada & 2,0 & 3,6 & 9,8 & 5,9 & 0,7 & 22 \\
\hline TOTAL & 13,2 & 18,3 & 36,2 & 31,1 & 1,2 & 100 \\
\hline
\end{tabular}

Fuente: Elaboración propia.

13 Manuel Castells desde hace años, en varios trabajos, planteó este momento como una era de la información, en donde el Internet funge como una de las principales vertientes en la producción de capital, tanto en la organización como en la democratización de ciertos procesos políticos (Castells, 2001). 
A pesar de que se podría inferir que en las universidades públicas exista una visión de perspectiva crítica de los medios y de la información tradicional $\mathrm{u}$ oficial ${ }^{14}$, se observa un comportamiento similar a nivel general en ambos contextos universitarios. Este grupo de ideas podrían utilizarse para grupos más cerrados o facultades por ejemplo, pero a nivel general el comportamiento indica una no distinción por universo público o privado de universitarios.

No puede dejar de mencionarse que, dentro del análisis de La Nación - a diferencia de Canal 7 por ejemplo- existe un sesgo ideológico que ubica al periódico en una corriente liberalconservadora más marcada, lo que implica que sus publicaciones sean evaluadas $y$ en muchos casos desacreditadas desde este prisma con mayor regularidad por parte de población universitaria. Esto es señalado en diversos estudios, particularmente en universidades públicas (Carballo, 2011).

Por otra parte, con respecto al medio televisivo, 54,4\% de los universitarios encuestados calificaron a Teletica Canal 7 como un medio "poco o nada" confiable (véase Cuadro 7). Esto es un dato importante, pues este canal ha liderado históricamente los estudios de audiencia, pero en este caso se enfrenta a una situación en la cual la población universitaria ha mostrado poca credibilidad por diversas situaciones $y$ ha reevaluado sus fuentes de información, como podría ser por ejemplo el Internet y la diversidad con la que puede contar esa plataforma.

Se resalta con respecto a los datos desagregados que la población estudiantil de las universidades públicas se ubicó mayormente en una "poca confiabilidad" $(27,7 \%)$, pero en el caso de la población estudiantil de las universidades privadas, el mayor porcentaje se ubicó en una confiabilidad "suficiente" $(9,8 \%)$. Es decir, a diferencia de lo ocurrido con $L a$ Nación, para el caso del medio de televisión Teletica Canal 7 la mayor confianza está ubicada en el sector de educación universitaria privada, mientras que la confianza disminuye si se encuentra en el espectro de educación universitaria pública.

\section{CUADRO 7 \\ CONFIABILIDAD DEL MEDIO TELEVISIVO TELETICA CANAL 7 SEGÚN LA PERCEPCIÓN DE LA POBLACIÓN ESTUDIANTIL UNIVERSITARIA SONDEADA 2015}

\begin{tabular}{lcccccc}
\hline \multicolumn{7}{c}{ PORCENTAJES } \\
\hline UNIVERSIDAD & NADA & POCO & SUFICIENTE & BASTANTE & NS/NR & TOTAL \\
\hline U. Pública & 17,4 & 27,7 & 22,3 & 8,8 & 2,0 & 78,2 \\
U. Privada & 2,9 & 6,4 & 9,8 & 2,0 & 0,7 & 21,8 \\
TOTAL & 20,3 & 34,1 & 32,1 & 10,8 & 2,7 & 100 \\
\hline
\end{tabular}

Fuente: Elaboración propia.

14 Sobre esta afirmación se necesita ampliar las limitantes y consideraciones, que van desde la carrera que se estudia hasta las cercanías con grupos políticos o trayectorias familiares. 
La presunción explicativa podría indicar que la visión crítica de las universidades públicas fomenta un distanciamiento del medio televisivo (contando también el tema de la carrera que se estudia y su contenido analítico $y$ posiciones paradigmáticas), mientras que acerca de la visión del grupo estudiantil de educación universitaria privada no verían en dicho cuestionamiento un interés o una necesidad de señalamiento marcada. Inclusive, puede que haya similitudes discursivas o coincidencias en las miradas.

Cabe comparar este criterio en el cual la población estudiada se aleja o cuestiona una empresa mediática tradicional, que es a su vez un referente importante de la plataforma que según su criterio fue la más influyente en la decisión de voto de la población costarricense en general. Esta contradicción es un hallazgo importante respecto a los procesos de comprensión que intervienen en la relación entre medios de comunicación, confiabilidad e impacto político. En otras palabras, colisionan dos ideas: por un lado, la percepción de que la televisión es la que mayor peso tiene sobre la influencia política y por otro, la desconfianza sobre un representante mediático de la misma televisión (al menos en este caso) que condensa la presencia mediática histórica de televisión en Costa Rica (con los respectivos matices analíticos).

\section{CONCLUSIONES ANALÍTICAS}

Esta investigación inició preguntándose sobre la percepción en la influencia mediática de los medios de comunicación - televisión e Internet-, en la población universitaria costarricense entre los 18 y 30 años en el marco del periodo político electoral 2014. Con ello se pretendió ahondar en las percepciones y prácticas de una población de características específicas como lo es la universitaria.

En términos generales se desprenden tres principales conclusiones asociadas a los tres puntos centrales del desarrollo analítico; pero dos principales elementos del presente artículo subyacen: por un lado, la evidencia empírica, según el sondeo realizado y por otro lado, la reflexión sobre un mundo centralizado en el entorno universitario. Así entonces, se plantea una primera conclusión de carácter descriptivocontextual; una segunda está asociada a plataformas de legitimidad político-comunicativas y por último, una reflexión sobre medios específicos y su visión de confiabilidad. Cabe indicar el factor especulativo que se le ha impreso a este artículo, ya que es una investigación exploratoria que busca ser un punto de encuentro para la discusión, cotejo y distanciamiento si fuera lo que amerita.

a) Conclusión descriptivo-contextual: la primera conclusión hace alusión a los datos sondeados en el nivel de la tendencia de votos en la primera y segunda ronda en la elección presidencial costarricense del año 2014. Lo indicado por los universitarios refleja el patrón de disminución en la segunda ronda, respecto de la primera, como también se vio en los datos de votación totales. Sin embargo, los datos de la población sondeada indican más grado de participación electoral. Esto puede deberse principalmente a: 1) Una combinación señalada de novedad, tanto vivencial (primera vez que se realiza el voto) como de los candidatos (aparición de otros candidatos "distintos" y no tradicionales). 2) Contexto universitario (principalmente en el ámbito de universidades públicas) que estimulaba el acercamiento en algún factor o dimensión política. Así, el contexto universitario es un estimulante fundamental en la generación de iniciativa política, lo cual reafirma la trayectoria histórica de participación social, sobre todo en el entorno público.

b) Conclusión sobre plataformas de legitimidad político-comunicativa: en el plano del uso de medios de comunicación y sus respectivas plataformas tecnológicas se afirma, de acuerdo a los datos, que la televisión mantiene la cumbre del entorno comunicacional que más influye sobre la política nacional a partir de los universitarios sondeados. En segundo lugar, ubicaron Internet, reafirmando el patrón mundial de asimilación en el 
entorno socio-político de esta plataforma comunicativa, lo cual era esperable. Que la televisión presente esta centralidad hace alusión a la tradicionalidad y facilidad de acceso que presenta todavía, además de albergar la mayor cantidad de pauta política en las elecciones en general. Sin embargo, se comparte que la resonancia de ambos - televisión e internet- (con las consideraciones analíticas que se habría que hacer) todavía no da para hablar de un contexto de divergencia marcada o de migración endógena y cerrada entre plataformas. Para el caso de la población estudiada son complementarias en el entorno político (al menos el convencional).

c) Conclusión sobre medios privados $y$ confiabilidad: la histórica visión de La Nación como un medio con una tendencia liberal-conservadora desde su fundación tiene una influencia general de confiabilidad "suficiente" en la población universitaria sondeada. Sin hacer énfasis en facultades o carreras con contenido más político o ideológicamente marcado, los datos generales indican una asociación entre fuerza política y asimilación social con dicho medio. En el caso de Teletica Canal 7, si bien tiene una cobertura muy alta dentro del medio de información más accesado, su credibilidad (sobre todo para los estudiantes de las universidades públicas) tiende a bajar. Como se indicó, esta es una contradicción en tanto la plataforma de más influencia política es la televisión, a pesar de que un representante de dicho medio es a su vez el de menor confiabilidad (respecto al otro estudiado). Una razón para dicha contradicción resultaría en reconocer que la televisión y su facilidad para ser consumida tienen réditos electorales (entre otros como la comercialización, por ejemplo) y que pese a la desconfianza o cuestionamiento a su credibilidad (en el medio escogido, pero de mucho peso social) la mirada teledirigida sigue siendo un punto a mantener presente en los análisis.

\section{REFERENCIAS}

Araya, J. (6 de julio de 2016). Alto costo frena acceso a Internet de alta velocidad en países de la región. Semanario Universidad. Recuperado de https:// semanariouniversidad.com/pais/ alto-costo-frena-acceso-internet-altavelocidad-paises-la-region/

Carballo, P. (2011). Análisis del discurso mediático sobre el sistema educativo público. Análisis del diario La Nación. Mayo 2007-abril de 2008. Revista de Ciencias Sociales, (133-134), 75-90.

Carballo, P. (2015). ¿Debatir o no Debatir? He ahí la cuestión. Una reflexión de la campaña electoral en Costa Rica: 20132014. Revista de Ciencias Sociales, (147), 27-48.

Carballo, P. (2016). Tendencias, fracturas y quiebres en la discursividad políticoelectoral costarricense. Análisis políticodiscursivo durante el proceso de la campaña presidencial 2013-2014. Revista de Ciencias Sociales, (153), 147-171.

Caro, A. (1994). La publicidad que vivimos. Madrid: Editorial Eresma Y Celeste Ediciones.

Castells, M. (2011). Internet y la sociedad red. Lección inaugural del programa de doctorado sobre la sociedad de la información y el conocimiento. Universitat Oberta de Catalunya (uoc). Recuperado de: http://www.uoc.edu/web/ cat/articles/castells/castellsmain2.html

Ceciliano, Y. (2008). Perspectivas juveniles en Costa Rica. San José, Costa Rica: flacso.

D'Adamo,O. J.; García, V. y Freidenberg, F. (2000). Efectos políticos de los medios de comunicación. Un análisis de la función del establecimiento de la agenda. Psicología política, (20), 47-63. Recuperado de http://www.uv.es/garzon/ psicologia\%20politica/N20-3.pdf

De Gori, E; Pirker, K y Villacorta, C. (comps.) (2014). Año de elecciones. El Salvador y 
Costa Rica: Miradas sobre el orden politico. Buenos Aires, Argentina: Sans Soleil.

Escalona, M. A. (2012). De cuando los imaginarios protegen del conflicto: La Costa Rica imaginada durante la polarización ante el TLc. Anuario Centroamericano de Costa Rica, 38, 329-354

Gómez, M. (2012). Elementos de estadística descriptiva. San José, Costa Rica: euned.

Instituto Nacional de Estadísticas y CensosINEC (2015). Tecnologías de información y comunicación en hogares. Costa Rica. Recuperado de http://www.inec. go.cr/otros-temas-ciencia-y-tecnologia/ tecnologias-de-informacion-y-comunicacion-en-hogares

Jiménez, G. (2013). La guerra de los medios y la oportunidad digital en Costa Rica. Recuperado de http://www.culturacr. net/14/05/La-guerra-de-los-medios-yel-sismo-digital-en-Costa-Rica.html\#. V4Fb4tLhBH3

Klingemann, H. (2004). La orientación de valores y la participación política en los Estados Bálticos. En Díez, N. e Inglehart, R. (comps.) Tendencias mundiales de cambio en los valores sociales y políticos (pp.405-422). Madrid: Fundesco.

Mora, S. (2008). Diez años de acciones colectivas en Costa Rica. Revista Centroamericana de Ciencias Sociales, V, (1), 131-168.

Murga, A. (2009). La participación política de los estudiantes universitarios en el primer gobierno de alternancia en México. Razón y Sociedad, (45), 45-63.

Nicolás, Y.; Alvarado, E.; Rodríguez, J. y Abarca, A. (2015). Las promesas electorales de los candidatos a la presidencia de la República de Costa Rica, divulgadas en medios de prensa escrita, 2014. Revista de Ciencias Sociales, (147), 15-26.

Ocampo, Á. (2010). Conocimiento y política en las universidades latinoamericanas. San José, Costa Rica: Editorial ucr.

osal. (2012). Movimientos Estudiantiles. Observatorio Social de América Latina.
Núm 31 (año xIII). Buenos Aires, Argentina: CLACSO.

Pignataro, A. y Treminio, I. (2015). Jóvenes y democracia: Comportamiento electoral y actitudes políticas en Costa Rica. Revista de Derecho Electoral, (20), 309-343.

Programa Estado de la Nación en Desarrollo Humano Sostenible. (2015). Estado de la Educación Costarricense. Informe 5. Programa Estado de la Nación en Desarrollo Humano Sostenible. San José, Costa Rica.

Raventós, C; Fournier, M; Fernández, D; y Alfaro, R. (2012). Saluda, voz y lealtad. Respuestas ciudadanas ante el malestar con la política. San José, Costa Rica: Universidad de Costa Rica y Instituto de Formación y Estudios en Democracia.

Salas, O. (2013). Redes sociales y campaña política. Recuperado de https://www.ucr. ac.cr/noticias/2013/11/06/redes-socialesy-campana-politica.html

Solano, J. (2001). Educación y desarrollo en América Latina. Un análisis históricoconceptual. Heredia, Costa Rica: euna.

Solís, L.P. (2014). Costa Rica en el siglo xxi: La que dejó de ser y la que no termina de nacer. De Gori et al. (comps.), En Año de elecciones. El Salvador y Costa Rica: Miradas sobre el orden político: 74-85. Buenos Aires: San Solei.

Tribunal Supremo de Elecciones de Costa Rica. (2014). Elecciones generales en cifras 1953-2014. [En línea]. Recuperado de http://www.tse.go.cr/pdf/elecciones/ eleccionescifras.pdf

Villalobos, N. (13 de enero de 2016). Principal medio informativo de los ticos es la televisión, según encuesta ucr. El País Digital. Recuperado de http://www. elpais.cr/2016/01/13/principal-medioinformativo-de-los-ticos-es-la-televisionsegun-encuesta-ucr/

Fecha de ingreso: $21 / 11 / 2016$ Fecha de aprobación: 27/04/2017 
